\title{
Review Article \\ Network Pharmacology: A New Approach for Chinese Herbal Medicine Research
}

\author{
Gui-biao Zhang, ${ }^{1}$ Qing-ya Li, ${ }^{1,2}$ Qi-long Chen, ${ }^{1}$ and Shi-bing Su${ }^{1}$ \\ ${ }^{1}$ Research Center for Traditional Chinese Medicine Complexity System, Shanghai University of Traditional Chinese Medicine, \\ Shanghai 201203, China \\ ${ }^{2}$ Henan University of TCM, Zhengzhou 450008, China \\ Correspondence should be addressed to Shi-bing Su; shibingsu07@163.com
}

Received 26 January 2013; Revised 28 March 2013; Accepted 2 May 2013

Academic Editor: Wei Jia

Copyright (C) 2013 Gui-biao Zhang et al. This is an open access article distributed under the Creative Commons Attribution License, which permits unrestricted use, distribution, and reproduction in any medium, provided the original work is properly cited.

\begin{abstract}
The dominant paradigm of "one gene, one target, one disease" has influenced many aspects of drug discovery strategy. However, in recent years, it has been appreciated that many effective drugs act on multiple targets rather than a single one. As an integrated multidisciplinary concept, network pharmacology, which is based on system biology and polypharmacology, affords a novel network mode of "multiple targets, multiple effects, complex diseases" and replaces the "magic bullets" by "magic shotguns." Chinese herbal medicine (CHM) has been recognized as one of the most important strategies in complementary and alternative medicine. Though CHM has been practiced for a very long time, its effectiveness and beneficial contribution to public health has not been fully recognized. Also, the knowledge on the mechanisms of CHM formulas is scarce. In the present review, the concept and significance of network pharmacology is briefly introduced. The application and potential role of network pharmacology in the CHM fields is also discussed, such as data collection, target prediction, network visualization, multicomponent interaction, and network toxicology. Furthermore, the developing tendency of network pharmacology is also summarized, and its role in CHM research is discussed.
\end{abstract}

\section{Introduction}

Over the past decades, drug discovery has followed the dominant paradigm of the "one gene, one drug, one disease" and mainly focused on designing exquisitely selective ligands which could avoid side effects [1]. However, owing to the lack of efficacy and safety, the clinical attrition rate of new drug candidates reached up to $30 \%$ [2]. Moreover, the largescale functional genomics studies have revealed that many single-gene knockouts exhibit little effect on the phenotype [3], and only $34 \%$ of single-gene knockout resulted in sickness or lethality [4]. Systems biology is a recent trend in bioscience research which focuses on the complex interactions in biological systems from a holistic perspective, rather than altering the single molecular component $[5,6]$. Network pharmacology $[7,8]$, a system biology-based methodology, replaces the corollary of rational drug design of "magic bullets" by the search for multitarget drugs that act on biological networks as "magic shotguns" [9]. Network biology analysis has also revealed that the deletion of individual nodes has little effect on the disease networks [10]. The increased understanding of the role of network biology systems challenges the dominant assumption of single-target drug discovery [11, 12]. Chinese herbal medicines (CHM) include natural medicines that were discovered by the ancient Chinese and evolved through at least 3000 years of uninterrupted clinical practice. Generally, $\mathrm{CHM}$ cures diseases by the synergistic effects of multiple compounds and herbal formula, which is mainly based on the integrative and holistic ways [13]. However, with the growing popularity and great promise of CHM, the everincreasing demand for illuminating pharmacological mechanisms, potential drug efficacy, and clinical toxicity are major issues that need to be addressed. As a methodology and technology, network pharmacology offers a new approach to integrate the notion of drug discovery based on comprehensive research and synthetic assessment. Obviously, this 
principle coincides with the characteristics of syndrome differentiation by traditional Chinese medicine (TCM) and holistic view of CHM treatment [14].

In this review, the concept and significance of network pharmacology is briefly introduced. Its application and potential role in CHM research is also summarized.

\section{Concept and Significance of Network Pharmacology}

With the rapid growth of available biomedical data in the postgenomic era, systems biology and polypharmacology have provided fresh insight into the drug discovery $[15,16]$. The computational biology provides profitable approach to address the scientific suspense through efficacious modeling and theoretical exploration. In 2007, Hopkins created a novel concept of network pharmacology, which is built on the fundamental concept that many effective drugs in therapeutic areas act on multiple rather than single targets [7,9]. Network pharmacology can be reconstructed with molecular networks that integrate multidisciplinary concepts including biochemical, bioinformatics, and systems biology [8]. It affords a rewarding assistance to forecast the off-target effects at a higher efficiency, which could improve the potency for drug discovery through a novel network mode of "multiple targets, multiple effects, complex diseases" [17].

The advantages of network pharmacology include the following: regulation of the signaling pathway with multiple channels, increase in drug efficacy, reduction of side effects, increase in the success rate of clinical trials, and decrease in the costs of drug discovery. Many complex diseases involve the interactions of multiple genes and functional proteins [18]. Network pharmacology models aim at addressing questions such as how and where in the disease network one target inhibits or activates the disease phenotypes. This ideally leads to therapies that are less vulnerable to drug resistance and lesser side effects by means of attacking the disease network at the systems level through synergistic and lethal interactions. The drug discovery strategies thereby should explore the regulated pathology network and reduce the typically high attrition rates in the disease networks [19]. Many studies have reported the interesting biological findings from these networks, and more than $40 \%$ of the drug-activated targets were discovered based on the disease gene networks by meta-analysis [20], which were associated with a number of diseases [21]. Therefore, network pharmacology can assist in systematic characterization of drug targets, thereby helping to decrease the high failure rates in discovery projects.

\section{Research Approaches of Network Pharmacology}

Network pharmacology can make an impact at two main approaches in the drug development process. One is to establish a pragmatic network model and predict the drug target based on public databases or available data of earlier researches. Subsequently, the mechanism of functional drug should be explored for the network equilibrium principle.
Based on this approach, $\mathrm{Gu}$ et al. calculated the effect of Rheidin A and C and Sennoside C, which was the first report on multiple component drugs for type II diabetes [22]. Yan et al. also predicted the new pharmacological action of Ephedra Decoction using virtual screening and network forecast techniques [23].

The other approach is to reconstruct a "drug target disease" network prediction model using the high-throughput screen (HTS) technology and bioinformatics methods. In this approach, the mechanism of drugs in the biological network was analyzed by comparing the interaction between the drug and the model. Many examples on the application of network pharmacology in drug discovery have been reported in the literature. Li et al. [24] used Liuwei Dihuang pill (a CHM formula) to predict the suitable network targets in disease treatment and found that the multilayer networks may underlie the combined mechanisms of herbal formula. Furthermore, nine components were screened in Fufang Danshen formula based on network pharmacology, which could modulate 42 cardiovascular-associated genes [25]. Additionally, by integrating the above research approaches, it was demonstrated that salvianolic acid B was compatible and feasible for cardiovascular disease treatment [26].

\section{Process in Network Pharmacology Research}

4.1. Data Collection and Validation. Network pharmacology takes into account the aforementioned principles to optimize the efficacy and safety of a candidate drug and their potent combinations. These also represent the two important steps in any experimental study. The first step of network pharmacology is the selection of original data from the experiments to build a biological network. The second is the experimental validation for the predicted network model. The validated data can be quantified using many different integrated methods including genomics, proteomics, metabolomics, and HTS/high-content screening (HCS) technologies [27].

HTS/HCS technologies can rapidly detect millions of data samples, identify substances, and modulate a particular molecular pathway or alter the phenotype of a cell $[28,29]$. These have many desirable features such as homogeneous, multidimensional phenotypic detection, real-time, dynamic monitoring, and visualization. Moreover, this dual highthroughput technology also can collect network data from the experiments and validate the network model. For instance, Fakhari and Dittmer created the polymerase chain reaction (PCR) chip technology to detect the gene expression [30]. The results demonstrated that the technology was convenient for the high-throughput studies.

Molecular interaction validation technology is another tool which validates the approach for network pharmacology, reveals the drug activity mechanisms, and verifies the drug network or predicted model. It can help researchers to discover the relationship between the drug and the macromolecules, and it mainly includes surface plasmon resonance (SPR) [31] and biolayer interferometry (BLI) technologies [32]. All of these techniques involve high-throughput, highprecision, label-free, and real-time detection. 
4.2. Network Analysis and Visualization. Network analysis focuses the on established network using related technology and extracts useful information which is convenient for further studies [33]. Three types of network analysis are available. The first one involves the calculation of the optimal topological structure and statistical properties of network after the extraction of specific network data [34], which is conserved as the hidden information in the network maximally. Secondly, the generation and comparison of random networks is used to check the reliability of the existing network by inducing acceptable modulation [35]. At last, the hierarchical clustering of the network is performed [36], algorithm is applied to predigest the complicated network, and potential information in the network is anticipated.

Network visualization is applied to extract the interaction information from interassociation data and switch them into a visual network using visualization tools [33]. This process contains two steps: (1) enriching network attributes, adding network nodes, and increasing connection power of network; (2) describing the network and taking abundant instrument to describe the architectural feature that clearly and intuitively represents the network. At present, most visualization of network pharmacology is through professional tools such as Cytoscape [37], GUESS [38], and Pajek [39]. Brief information of network pharmacological technologies and tools is shown in Table 1.

\section{Application of Network Pharmacology in CHM Research}

CHM, the ancient treatment methodology popular in China and surrounding areas, has been recognized as a pharmaceutical area of TCM and holds promise for preventing diseases in a holistic way $[40,41]$. In a long period of clinical practice, it is known for its effectiveness and beneficial contribution to public health and disease control. However, the pharmacological mechanisms of CHM have not been fully established. With increasing knowledge of the network of genes and molecular interactions, the researchers adopt network pharmacology for their drug research and development. Figure 1 shows the developing tendency of network pharmacological studies from the data available in Web of Knowledge, PubMed, and China National Knowledge Infrastructure (CNKI) databases from 2007 to 2012. The applications of network pharmacology in CHM were systematically summarized to demonstrate the significant value in this area of research.

5.1. Construction and Application of CHM Database. Building a CHM database is critical for a network pharmacology study. Chen et al. [42] constructed the TCM-ID database including TCM prescriptions, herbal ingredients, and 3D structure of herbal ingredients. It was mainly used for illustrating the mechanism of the effects of herbal ingredients. Ye et al. [43] integrated the text-mining technology, a strict artificial audit and annotation process, and obtained the protein targets of Chinese medicine by determining the effective components based on the mass literature from PubMed.

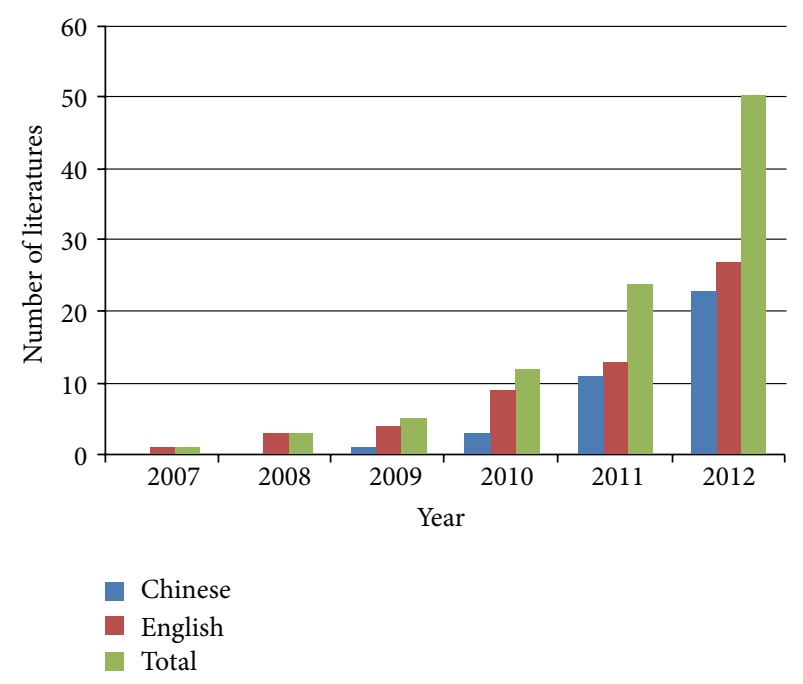

FIGURE 1: Developing tendency of network pharmacology study. The publications of network pharmacology study in Web of Knowledge, PubMed, and CNKI databases from 2007 to 2012. All results were screened in manual way.

Subsequently, the Herbal Ingredients' Targets Database provided the integrated information for the Chinese herbal active ingredient protein target and offered platform to analyze the similarity between the compound formula and protein sequence.

Furthermore, many other TCM databases have been established for network pharmacology research, such as the TCMGeneDIT database [44] and TCM Database@Taiwan [45]. The former mainly focuses on TCM-related gene and disease information, and the latter is applied to CHM screening. In addition, the disease-drug-target databases such as SuperTarget, Matador [46], DrugBank [47], and Therapeutic Target Database [48] are also used for drug-target research on herbal compounds.

5.2. Predictions of CHM Drug Target. Relevant technology can be used to screen the effective herbal substances and discover the drug target. Such technology could also provide theoretical support for detecting new pharmacological effects of Chinese compound formula. Li [49] proposed a methodology termed "network target," which is used to reveal the interactions between herbal compounds/formulas and complex syndrome systems based on network pharmacology and systems biology. Wu et al. [50] predicted the multitarget of Aconiti Lateralis Radix Praeparata's multi-compound based on the drug-target data and random forest algorithm. They found that each compound was correlated with 16.3 targets, whereas each target was related to 4.77 compounds. This study reflects the notion of "multi-compound and multitarget" in CHM towards drug discovery.

Yu et al. [51] predicted the new pharmacological effects (antihyperglycemic and antihyperlipidemic) of Fuzheng Huayu Capsule using high-throughput technology and Connectivity Map databases [52]. Using the chemical structure of compounds, target identification, and enrichment analysis, 
TABLE 1: Brief introduction of network pharmacological technologies methods and tools.

\begin{tabular}{|c|c|c|c|}
\hline \multicolumn{4}{|c|}{ Mainly experimental techniques and tools in network pharmacology } \\
\hline Technique & Application fields & Advantage & Literatures \\
\hline HTS/HCS & Massive data acquisition & $\begin{array}{l}\text { Homogeneous, multidimensional phenotypic detection, } \\
\text { dynamic real-time monitoring, and visualization }\end{array}$ & {$[28,29]$} \\
\hline PCR chip & Massive data acquisition & $\begin{array}{l}\text { Dual high throughput, strong specificity, high sensitivity, and } \\
\text { good repeatability }\end{array}$ & {$[30]$} \\
\hline SPR & Massive data acquisition & $\begin{array}{l}\text { No marks, high-throughput, high-precision, and real-time } \\
\text { detection }\end{array}$ & {$[31,32]$} \\
\hline BLI & Massive data acquisition & $\begin{array}{l}\text { No marks, high-throughput, high-precision, and real-time } \\
\text { detection }\end{array}$ & {$[31,32]$} \\
\hline Cytoscape & Network visualization & $\begin{array}{l}\text { Graphic operation, construct simple network, plugin support } \\
\text { for analysis, and easy to use }\end{array}$ & [37] \\
\hline GUESS & Network visualization & $\begin{array}{l}\text { Graphic operation, command line, and script support for } \\
\text { analysis }\end{array}$ & [38] \\
\hline Pajek & Network visualization & Graphic operation, building large-scale network & [39] \\
\hline $\begin{array}{l}\text { Network topology } \\
\text { information calculation }\end{array}$ & Network analysis & Classify and sequence the nodes, reflect hidden information & {$[34]$} \\
\hline $\begin{array}{l}\text { Random network creation } \\
\text { and comparison }\end{array}$ & Network analysis & Verify reliability of existing network & {$[35]$} \\
\hline $\begin{array}{l}\text { Network layer and } \\
\text { clustering }\end{array}$ & Network analysis & $\begin{array}{l}\text { Simplify the complexity of network, find out potential } \\
\text { information }\end{array}$ & {$[36]$} \\
\hline
\end{tabular}

Zhu and Yao [53] successfully predicted the molecular targets of Xiaochaihu Decoction. They found that the potential targets of the 21 compounds mainly involved metabolic, inflammation, and poison degradation processes. Zhang et al. [54] created a new algorithm to predict the molecular targets of rhein, which integrated the protein-protein interactions, pathways, genome expression, and the literature data mining. The results showed that three specific genes were relevant for drug targets, and their functions mainly involved cellular apoptosis, immunity, and transport.

\subsection{Network Visualization of the CHM Literature Mining} Researches. Essentially, the network visualization of the CHM literature examines the database to find modes or rules [55], detects the literature information, analyzes the selection data, and discovers the novel effects of CHM. For example, Li et al. [56] integrated the microarray and the literature database to design a literature mining and microarray analysis system, which was used to construct biological networks and reveal the related interactions.

Wu et al. [57] collected slices information of TCM from the Chinese Pharmacopoeia by text mining and constructed the TCM slices-symptom network. They discovered 3016 pairs of TCM slice-symptom correlations. Each TCM slice was correlated with 4.67 symptoms, and each symptom was related to 7.47 TCM slices. Furthermore, the network analysis also indicated that network pharmacology approaches could be used to forecast and discover unknown or new effects and channel tropisms of TCM slices. Additionally, Zhang et al. [58] reconstructed three Bayesian belief networks [59] for bitter, sweet, and pungent flavors, which was based on the modern pharmacology of TCM slices and clinical data. The results suggested that the Bayesian belief network could be used to predict the flavors of Chinese medicinal components and perform further studies of drug properties and drug compatibility.

5.4. CHM Multicomponent and Formula Researches. In order to discover the relationship between Chinese herbal multicomponent and potential pharmacological function, Li et al. [60] applied a network target-based identification of multicomponent synergy (NIMS) algorithms to calculate the component of $\mathrm{CHM}$ and demonstrate the synergistic correlation of the multicomponent. The results proposed that the NIMS approach could be beneficial for analyzing the therapeutic effects of CHM multicomponent. In the Tougu Xiaotong capsule (TGXTC) study, Zheng et al. [61] analyzed 514 components using network pharmacology and computational pharmacological methods. By analyzing the network parameters of the TGXTC compound-target and drug-target networks, they revealed the molecular mechanism of multicomponent, multitarget, and multipathway in TGXTC.

Zhu et al. [62] used molecular docking and complex analytical techniques to study the pharmacodynamic function of multicomponent in spleen-regulating heart-nourishing formula. They found that the scale-free feature and node attribute of networks clearly illuminated the pharmacological function of CHM multicomponent. Exhilaratingly, some recent studies revealed the mechanisms of multicomponent efficiency in CHM for the treatment of cardiovascular disease $[63,64]$ by systematic investigation, which have constructed "CHM components targets" networks based on chemical 
components, chemical structures, chemogenomics, and target predictors data.

5.5. CHM Network Toxicology Researches. Network toxicology is based on comprehension of "toxicity (side effects) gene target drug," which utilizes the network analysis to speculate and estimate toxicity and side effects of drugs. It focuses on the toxic reaction of specific component in a complex system and provides assistance for drug safety evaluation and research. The study of Liu et al. [65] identified that the integrated high-throughput biochip technologies and drug-target network could afford significant values for activated ingredient screening, toxic components exclusion, and molecular mechanisms of CHM research. Fan et al. [66] used network pharmacology method to reconstruct the network model to describe the toxicological properties, which offered valuable information to identify the toxic substances and potential toxicity of known compounds in a complex system. Zhou et al. [67] analyzed the nephrotoxicity of aristolochic acid based on the metabolic network and established an integral and dynamical progression of drug toxicity method.

In addition, the databases such as Comparative Toxicogenomics Database, National Toxicology Program, and Toxicology Data Network are widely used for network toxicology studies $[68,69]$. Furthermore, the forecasting toxicity software such as Toxicity Prediction by Komputer-Assisted Technology, HazardExpert, DEREK, and Prediction System of Carcinogenic Toxicity [70] is other available tools for CHM network toxicology studies.

5.6. TCM Syndrome-Based Network Pharmacology Researches. The feature of TCM is based on the syndrome differentiation, which emphasizes the integrating disease and syndrome. Monarch, minister, assistant, and guide in TCM prescription contain many principles of system theory, and the aim of coordination and cooperation of several kinds of CHM is to regulate body functional imbalances and disorders. Therefore, network pharmacology could be used for TCM diagnosis based on "disease-syndromeformula" model, which integrates the information of "diseasephenotype-gene-drug" and builds a "disease phenotype biological molecule" network [24]. In a study on patients with rheumatoid arthritis (RA), Niu et al. [71] revealed the molecular mechanism of "herbs-pattern correspondence" with heat pattern in TCM, which analyzed the drug-target molecular network. Four common canonical pathways were found to be involved in these: GM-CSF signaling, CLTA4 signaling in cytotoxic T lymphocytes, T-cell receptor signaling, and CD28 signaling in T-helper cells. These uniform pathways implicated that the "herbs-pattern correspondence" could more likely be associated with heat pattern of RA.

ZHENG is a complex concept in TCM. Li et al. [72] reconstructed the neuroendocrine-immune (NEI) network by systems biology approach combined with animal experiments. The results showed that the hormones were predominant in the Cold ZHENG network, whereas immune factors were predominant in the Hot ZHENG network. In particular, two networks were connected by neurotransmitters, which suggested that ZHENG might have a special molecular mechanism from the background of NEI study. Using the microarray samples of liver-gallbladder dampnessheat syndrome and liver depression and spleen deficiency syndrome in chronic hepatitis B and liver cirrhosis, Guo et al. [73] elucidated the molecular mechanisms of the same TCM syndrome for different diseases and different TCM syndrome for the same disease, which might be related to the G-proteincoupled receptor protein-signaling pathway. Moreover, Shi et al. [74] used a complex network and chi-squared automatic interaction detector decision tree to identify the core syndromes of TCM in coronary heart disease (CHD) and establish TCM syndrome identification modes of CHD based on the four diagnostic information and biological parameters.

\section{Discussion and Conclusion}

Although TCM has a long history of clinical practice in China, it is considered as a complementary and alternative medicine in the rest of the world. The biggest obstacles of $\mathrm{CHM}$ development are the multiple in vivo pathways of $\mathrm{CHM}$ metabolites from their multiple drug components, which are essential for their pharmacological actions. However, the knowledge on the effective mechanisms of CHM formulas is scarce. Moreover, the CHM mechanism is considered as the synergistic effect of active drug ingredients and results of complex biological interactions. Fortunately, as a novel approach, network pharmacology takes into account the multidisciplinary and cross-disciplinary fields to optimize the efficacy and safety of drug discovery. Hence, researchers started utilizing it to study the drug targets and efficacy of CHM [75]. Network pharmacology has become a helpful tool to understand the details of drug-target, especially for multiple drug components of CHM.

Generally, network pharmacology of CHM has been considered to contain static and dynamic configurations. The static configuration is called a network pharmacy metrology with chromatographic fingerprint, while the dynamic is called a network pharmacodynamics with chromatographic fingerprint (NPDCF). The key problem for CHM formula network pharmacology is how to confirm the parameters of NPDCF by network equilibrium constants [76]. For example, He et al. [77] analyzed the multiple drug components from the parameters of network pharmacokinetic model and found that the effects of CHM formulas were inhibited by pharmacokinetic and pharmacodynamic coefficients [78]. In the static multicomponent studies of CHM formula, Tal et al. [79] predicted the active ingredients and potential targets of Chinese herbal Radix Curcumae formula using network pharmacology, which was used for the treatment of cardiovascular disease. However, the network dynamics research of CHM formulas need more evidence.

The characteristics of TCM theory involve the consideration of organic wholeness and treatment based on TCM syndrome differentiation. A diagram is proposed to exhibit the research approach of network pharmacology for CHM 


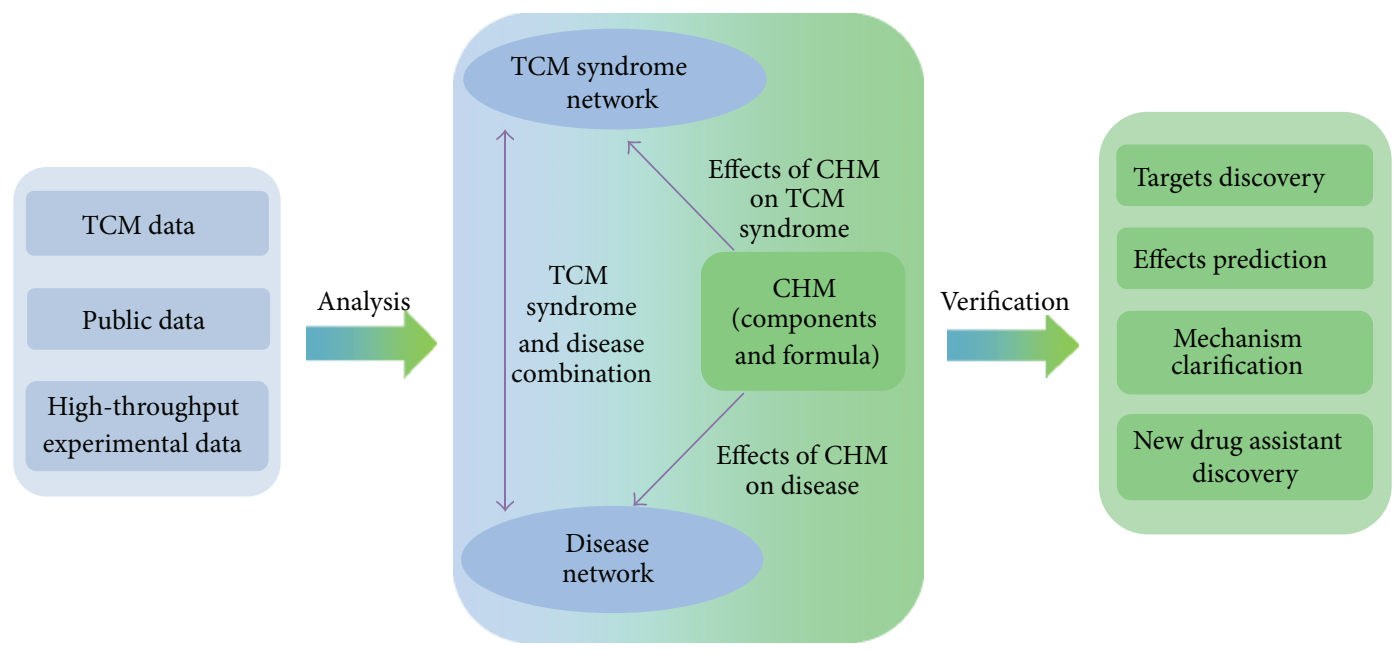

FIGURE 2: Network pharmacology approach for CHM research. For the discovery of CHM-derived targets, effect prediction, mechanism clarification, and new drug assistant discovery using network pharmacology approach. It analyzes the information from public data, highthroughput experimental data, and TCM data and constructs a "CHM-TCM syndrome disease" interaction network using technologies of network expansion, optimization, comparison, knockout, and addition. Finally, it carries out computational and experimental verifications.

(Figure 2). This approach is a combination from "diseasesyndrome-CHM" model, which comprises the core values for reflecting disease and TCM syndrome as well as correlates with CHM, TCM syndrome, and multitarget effects. By integrating the chemical predictor, target predictor, and network building, a system of TCM was constructed. It systematically revealed the potential mechanisms of TCM [80]. The appropriate cellular and animal models are conducive to evaluate the effectiveness of TCM [81-83], which could be used to verify the results of network analysis and mutual authentication. However, the systemic characterization is still unclear for the drug-target correlation of CHM. Network pharmacology could be helpful to confirm the effective ingredients and promote drug discovery of CHM.

Network pharmacology has become a helpful tool in understanding the fine details of drug-target interactions. Using network pharmacology to investigate CHM pharmacological effects and drug targets, attention should be paid to degree centrality [84], betweenness centrality [85], and bridging centrality [86]. Network-based tools for analyzing topology and especially dynamics have great potential to identify alternative targets for finding and developing multitarget drugs [87].

In summary, the advancements in systems biology and bioinformatics will make an operational shift from reductionism in favor of network pharmacology and will undoubtedly bring about a conceptual move in drug discovery and make a significant contribution to CHM modernization and globalization.

\section{Acknowledgments}

This study was supported by the National Science and Technology Major Project of China (no. 2012ZX10005001-004),
Leading Academic Discipline Project of Shanghai Municipal Education Commission (no. J50301 and no. 2012JW25), Shanghai Municipal Science and Technology Commission Project (12401900401), and E-institutes of Shanghai Municipal Education Commission (no. E 03008).

\section{References}

[1] F. Sams-Dodd, "Target-based drug discovery: is something wrong?” Drug Discovery Today, vol. 10, no. 2, pp. 139-147, 2005.

[2] I. Kola and J. Landis, "Can the pharmaceutical industry reduce attrition rates?" Nature Reviews Drug Discovery, vol. 3, no. 8, pp. 711-715, 2004.

[3] B. P. Zambrowicz and A. T. Sands, "Modeling drug action in the mouse with knockouts and RNA interference," Drug Discovery Today, vol. 3, no. 5, pp. 198-207, 2004.

[4] M. E. Hillenmeyer, E. Fung, J. Wildenhain et al., "The chemical genomic portrait of yeast: uncovering a phenotype for all genes," Science, vol. 320, no. 5874, pp. 362-365, 2008.

[5] H. Kitano, "Systems biology: a brief overview," Science, vol. 295, no. 5560, pp. 1662-1664, 2002.

[6] U. Sauer, M. Heinemann, and N. Zamboni, "Getting closer to the whole picture," Science, vol. 316, no. 5824, pp. 550-551, 2007.

[7] A. L. Hopkins, "Network pharmacology," Nature Biotechnology, vol. 25, no. 10, pp. 1110-1111, 2007.

[8] A. L. Hopkins, "Network pharmacology: the next paradigm in drug discovery," Nature Chemical Biology, vol. 4, no. 11, pp. 682690, 2008.

[9] B. L. Roth, D. J. Sheffer, and W. K. Kroeze, "Magic shotguns versus magic bullets: selectively non-selective drugs for mood disorders and schizophrenia," Nature Reviews Drug Discovery, vol. 3, no. 4, pp. 353-359, 2004.

[10] P. Csermely, V. Ágoston, and S. Pongor, "The efficiency of multitarget drugs: the network approach might help drug design," Trends in Pharmacological Sciences, vol. 26, no. 4, pp. 178-182, 2005. 
[11] C. G. Wermuth, "Multitargeted drugs: the end of the "onetarget-one-disease" philosophy?" Drug Discovery Today, vol. 9, no. 19 , pp. 826-827, 2004.

[12] C. T. Keith, A. A. Borisy, and B. R. Stockwell, "Multicomponent therapeutics for networked systems," Nature Reviews Drug Discovery, vol. 4, no. 1, pp. 71-78, 2005.

[13] G. A. Luo, Q. L. Liang, R. L. Zhang et al., "Study of chemical matteromics and prescription of traditional Chinese medicineand an analysis of material foundation of compound prescription Qingkailing," World Science and Technology, vol. 8, no. 1, pp. 6-15, 2006.

[14] Z. H. Liu and X. B. Sun, "Network pharmacology: new opportunity for the modernization of traditional Chinese medicine," Acta Pharmaceutica Sinica, vol. 47, no. 6, pp. 696-703, 2012.

[15] A. Friboulet and D. Thomas, "Systems biology—an interdisciplinary approach," Biosensors and Bioelectronics, vol. 20, no. 12, pp. 2404-2407, 2005.

[16] J. T. Metz and P. J. Hajduk, "Rational approaches to targeted polypharmacology: creating and navigating proteinligand interaction networks," Current Opinion in Chemical Biology, vol. 14, no. 4, pp. 498-504, 2010.

[17] J. H. Pan, "New paradigm for drug discovery based on network pharmacology," Chinese Journal of New Drugs and Clinical Remedies, vol. 28, no. 10, pp. 721-726, 2009.

[18] X. Wu, R. Jiang, M. Q. Zhang et al., "Network-based global inference of human disease genes," Molecular Systems Biology, vol. 4, pp. 1-11, 2008.

[19] Y. Chen, J. Zhu, P. Y. Lum et al., "Variations in DNA elucidate molecular networks that cause disease," Nature, vol. 452, no. 7186, pp. 429-435, 2008.

[20] M. A. Yildirim, K. I. Goh, M. E. Cusick, A. L. Barabási, and M. Vidal, “Drug-target network," Nature Biotechnology, vol. 25, no. 10, pp. 1119-1126, 2007.

[21] A. L. Barabási, N. Gulbahce, and J. Loscalzo, "Network medicine: a network-based approach to human disease," Nature Reviews Genetics, vol. 12, no. 1, pp. 56-68, 2011.

[22] J. Gu, H. Zhang, L. Chen et al., "Drug-target network and polypharmacology studies of a Traditional Chinese Medicine for type II diabetes mellitus," Computational Biology and Chemistry, vol. 35, no. 5, pp. 293-297, 2011.

[23] J. Yan, Y. Wang, S. J. Luo et al., “TCM grammar systems: an approach to aid the interpretation of the molecular interactions in Chinese herbal medicine," Journal of Ethnopharmacology, vol. 137, no. 1, pp. 77-84, 2011.

[24] S. Li, B. Zhang, D. Jiang, Y. Wei, and N. Zhang, "Herb network construction and co-module analysis for uncovering the combination rule of traditional Chinese herbal formulae," BMC Bioinformatics, vol. 11, no. 11, article S6, 2010.

[25] X. Li, L. Wu, X. Fan et al., "Network pharmacology study on major active compounds of Fufang Danshen formula," China Journal of Chinese Materia Medica, vol. 36, no. 21, pp. 2911-2914, 2011.

[26] C. Ma, Y. Yao, Q. X. Yue et al., "Differential proteomic analysis of platelets suggested possible signal cascades network in platelets treated with Salvianolic acid B," PLoS ONE, vol. 6, no. 2, Article ID e14692, 2011.

[27] X. R. Cheng, W. X. Zhou, and Y. X. Zhang, "Experimental techniques in network pharmacology," Chinese Journal of Pharmacology and Toxicology, vol. 26, no. 2, pp. 131-137, 2012.

[28] B. S. Edwards, T. Oprea, E. R. Prossnitz, and L. A. Sklar, "Flow cytometry for high-throughput, high-content screening,"
Current Opinion in Chemical Biology, vol. 8, no. 4, pp. 392-398, 2004.

[29] F. Miscevic, O. Rotstein, and X. Y. Wen, "Advances in zebrafish high content and high throughput technologies," Combinatorial Chemistry \& High Throughput Screening, vol. 15, no. 7, pp. 515521, 2012.

[30] F. D. Fakhari and D. P. Dittmer, "Charting latency transcripts in Kaposi's sarcoma-associated herpesvirus by whole-genome real-time quantitative PCR," Journal of Virology, vol. 76, no. 12, pp. 6213-6223, 2002.

[31] X. Guo, "Surface plasmon resonance based biosensor technique: a review," Journal of Biophotonics, vol. 5, no. 7, pp. 483-501, 2012.

[32] C. A. Wartchow, F. Podlaski, S. Li et al., "Biosensor-based small molecule fragment screening with biolayer interferometry," Journal of Computer-Aided Molecular Design, vol. 25, no. 7, pp. 669-676, 2011.

[33] L. H. Wu, Y. Wang, and X. Fan, “Tools for network pharmacology study: network visualization and network analysis," China Journal of Chinese Materia Medica, vol. 36, no. 21, pp. 29232925, 2011.

[34] L. Cagnolo, A. Salvo, and G. Valladares, "Network topology: patterns and mechanisms in plant-herbivore and hostparasitoid food webs," Journal of Animal Ecology, vol. 80, no. 2, pp. 342-351, 2011.

[35] K. V. Brinda, S. Vishveshwara, and S. Vishveshwara, "Random network behaviour of protein structures," Molecular Biosystems, vol. 6, no. 2, pp. 391-398, 2010.

[36] L. Hou, L. Wang, A. Berg et al., "Comparison and evaluation of network clustering algorithms applied to genetic interaction networks," Frontiers in Bioscience, vol. 4, pp. 2150-2161, 2012.

[37] C. T. Lopes, M. Franz, F. Kazi, S. L. Donaldson, Q. Morris, and G. D. Bader, "Cytoscape web: an interactive web-based network browser," Bioinformatics, vol. 26, no. 18, Article ID btq430, pp. 2347-2348, 2010.

[38] E. Adar and M. Kim, "Soft GUESS: visualization and exploration of code clones in context," in Proceedings of the 29th International Conference on Software Engineering (ICSE '07), pp. 762-765, May 2007.

[39] D. N. Wouter, M. Andrej, and B. Vladimir, Exploratory Social Network Analysis With Pajek, Cambridge University Press, Cambridge, Mass, USA, 2005.

[40] J. Xu and Y. Yang, "Traditional Chinese medicine in the Chinese health care system," Health Policy, vol. 90, no. 2-3, pp. 133-139, 2009.

[41] F. Cheung, “TCM: made in China," Nature, vol. 480, pp. S82S83, 2011.

[42] X. Chen, H. Zhou, Y. B. Liu et al., "Database of traditional Chinese medicine and its application to studies of mechanism and to prescription validation," British Journal of Pharmacology, vol. 149, no. 8, pp. 1092-1103, 2006.

[43] H. Ye, L. Ye, H. Kang et al., "HIT: linking herbal active ingredients to targets," Nucleic Acids Research, vol. 39, no. supplement 1, pp. D1055-D1059, 2011.

[44] Y. C. Fang, H. C. Huang, H. H. Chen et al., “TCMGeneDIT: a database for associated traditional Chinese medicine, gene and disease information using text mining," $B M C$ Complementary and Alternative Medicine, vol. 8, p. 58, 2008.

[45] C. Y. Chen, “TCM Database@Taiwan: the world's largest traditional Chinese medicine database for drug screening In Silico," PLoS ONE, vol. 6, no. 1, Article ID e15939, 2011. 
[46] S. Gunther, M. Kuhn, M. Dunkel et al., "SuperTarget and Matador: resources for exploring drug-target relationships," Nucleic Acids Research, vol. 36, pp. D919-D922, 2008.

[47] C. Knox, V. Law, T. Jewison et al., "DrugBank 3. 0: a comprehensive resource for 'omics' research on drugs," Nucleic Acids Research, vol. 39, no. 1, pp. D1035-D1041, 2011.

[48] F. Zhu, B. Han, P. Kumar et al., "Update of TTD: therapeutic target database," Nucleic Acids Research, vol. 38, no. 1, pp. D787D791, 2009.

[49] S. Li, "Network target: a starting point for traditional Chinese medicine network pharmacology," China Journal of Chinese Materia Medica, vol. 36, no. 15, pp. 2017-2020, 2011.

[50] L. Wu, X. Gao, L. Wang et al., "Prediction of multi-target of aconiti lateralis radix praeparata and its network pharmacology," China Journal of Chinese Materia Medica, vol. 36, no. 21, pp. 2907-2910, 2011.

[51] S. Yu, Z. Guo, Y. Guan et al., "Combining ZHENG theory and high-throughout expression data to predict new effects of Chinese herbal formulae," Evidence-Based Complementary and Alternative Medicine, vol. 2012, Article ID 986427, 8 pages, 2012.

[52] J. Lamb, E. D. Crawford, D. Peck et al., "The connectivity map: using gene-expression signatures to connect small molecules, genes, and disease," Science, vol. 313, no. 5795, pp. 1929-1935, 2006.

[53] W. Zhu and L. M. Yao, "Potential targets and molecular mechanisms of, "Xiaochaihu Decoction" by Metadrug software," Shanghai Journal of Traditional Chinese Medicine, vol. 45, no. 1, pp. 79-82, 2011.

[54] A. H. Zhang, S. Hui, Y. Bo et al., "Predicting new molecular targets for rhein using network pharmacology," BMC Systems Biology, vol. 6, p. 20, 2012.

[55] I. H. Witten and E. Frank, Data Mining Practical Machine Learning Tools and Techniques, China Machine, Beijing, China, 2005.

[56] S. Li, L. Wu, and Z. Zhang, "Constructing biological networks through combined literature mining and microarray analysis: a LMMA approach," Bioinformatics, vol. 22, no. 17, pp. 2143-2150, 2006.

[57] L. Wu, X. Gao, Y. Cheng et al., "Symptom-based traditional Chinese medicine slices relationship network and its network pharmacology study," China Journal of Chinese Materia Medica, vol. 36, no. 21, pp. 2916-2919, 2011.

[58] P. Zhang, J. Li, Y. Wang et al., "Bayesian belief network and its application in predicting the five flavors of Chinese medicinal components," Modernization of Traditional Chinese Medicine and Materia Materia-World Science and Technology, vol. 10, no. 5, pp. 114-117, 2008.

[59] R. Montironi, W. F. Whimster, Y. Collan, P. W. Hamilton, D. Thompson, and P. H. Bartels, "How to develop and use a Bayesian belief network," Journal of Clinical Pathology, vol. 49, no. 3, pp. 194-201, 1996.

[60] S. Li, B. Zhang, and N. Zhang, "Network target for screening synergistic drug combinations with application to traditional Chinese medicine," BMC Systems Biology, vol. 5, no. 1, article S10, 2011.

[61] C. S. Zheng, Z. C. Lin, H. F. Xu et al., "Pharmacological research on osteoarthritis treated by tougu xiaotong capsule," Journal of Fujian University of TCM, vol. 21, no. 1, pp. 43-47, 2011.

[62] Y. F. Zhu, Z. W. Xu, H. Q. Ao et al., "Pharmacodynamic research of spleen-regulating and heart-nourishing formula based on network features," Traditional Chinese Drug Research \& Clinical Pharmacology, vol. 23, no. 1, pp. 25-29, 2012.
[63] X. Wang, X. Xu, W. Tao et al., "A systems biology approach to uncovering pharmacological synergy in herbal medicines with applications to cardiovascular disease," Evidence-Based Complementary and Alternative Medicine, vol. 2012, 15 pages, 2012.

[64] B. Li, X. Xu, X. Wang et al., "A systems biology approach to understanding the mechanisms of action of Chinese herbs for treatment of cardiovascular disease," International Journal of Molecular Sciences, vol. 13, no. 10, pp. 13501-13520, 2012.

[65] Q. Liu, Z. Zhang, L. Fang et al., "Application of network pharmacology and high through-put technology on active compounds screening from traditional Chinese medicine," China Journal of Chinese Materia Medica, vol. 37, no. 2, pp. 134-137, 2012.

[66] X. Fan, X. Zhao, Y. Jin et al., "Network toxicology and its application to traditional Chinese medicine," China Journal of Chinese Materia Medica, vol. 36, no. 21, pp. 2920-2922, 2011.

[67] M. M. Zhou, P. Liu, W. Jia et al., "Evaluation of therapeutic effects of TCM based on metabolic variation," Modernization of Traditional Chinese Medicine and Materia Materia-World Science and Technology, vol. 8, no. 6, pp. 113-119, 2006.

[68] A. P. Davis, B. L. King, S. Mockus et al., "The comparative toxicogenomics database: update 2011," Nucleic Acids Research, vol. 39, no. 1, pp. D1067-D1072, 2011.

[69] G. H. I. Wolfgang and D. E. Johnson, "Web resources for drug toxicity," Toxicology, vol. 173, no. 1-2, pp. 67-74, 2002.

[70] A. Lagunin, D. Filimonov, A. Zakharov et al., "Computeraided prediction of rodent carcinogenicity by PASS and CISOCPSCT," QSAR and Combinatorial Science, vol. 28, no. 8, pp. 806810, 2009.

[71] X. Y. Niu, J. Li, C. Lu et al., "The molecular mechanism of 'Herbs-Pattern Correspondence' in RA with heat pattern based on network pharmacology," Chinese Journal of Experimental Traditional Medical Formulae, vol. 18, no. 8, pp. 299-303, 2012.

[72] S. Li, Z. Q. Zhang, L. J. Wu, X. G. Zhang, Y. D. Li, and Y. Y. Wang, "Understanding ZHENG in traditional Chinese medicine in the context of neuro-endocrine-immune network," IET Systems Biology, vol. 1, no. 1, pp. 51-60, 2007.

[73] Z. Guo, S. Yu, Y. Guan et al., "Molecular mechanisms of 'Same TCM Syndrome for Different Diseases' and 'Different TCM Syndrome for Same Disease' in chronic hepatitis B and liver cirrhosis," Evidence-Based Complementary and Alternative Medicine, vol. 2012, 9 pages, 2012.

[74] Q. Shi, H. H. Zhao, J. X. Chen et al., "Study on TCM syndrome identification modes of coronary heart disease based on data mining," Evidence-Based Complementary and Alternative Medicine, vol. 2012, Article ID 697028, 2012.

[75] G. H. Du, Y. H. Wang, R. Zhang et al., "Multi-target and multicomponent pattern, a superficial understanding of the action mechanism of Traditional Chinese Medicine," Modernization of Traditional Chinese Medicine and Materia Materia-World Science and Technology, vol. 11, no. 4, pp. 480-484, 2009.

[76] F. Y. He, K. W. Deng, W. L. Liu et al., "Study on theoretical and technologic key problems of network pharmacology and pharmacodynamics for the Chinese Materia Medica Formula," Pharmacology and Clinics of Chinese Materia Medica, vol. 28, no. 5, pp. 256-259, 2012.

[77] F. Y. He, H. H. Zhou, and J. Y. Luo, "Establishment of a network pharmacokinetic model for multiple component drugs and parameter analyses," Chinese Journal of Clinical Pharmacology and Therapeutics, vol. 12, no. 12, pp. 1321-1331, 2007. 
[78] F. He, K. Deng, H. Zou, Y. Qiu, F. Chen, and H. Zhou, "Study on differences between pharmacokintics and chromatopharmacodynamics for Chinese materia medica formulae," China Journal of Chinese Materia Medica, vol. 36, no. 2, pp. 136-141, 2011.

[79] W. Y. Tal, X. Xu, X. Wang et al., "Network pharmacologybased prediction of the active ingredients and potential targets of Chinese herbal Radix Curcumae formula for application to cardiovascular disease," Journal of Ethnopharmacology, vol. 145, no. 1, pp. 1-10, 2013.

[80] B. H. Li, X. Xu, X. Wang et al., "A systems biology approach to understanding the mechanisms of action of Chinese herbs for treatment of cardiovascular disease," International Journal of Molecular Sciences, vol. 13, no. 10, pp. 13501-13520, 2012.

[81] M. Wang, X. Zhang, X. Xiong et al., "Efficacy of the Chinese traditional medicinal herb Celastrus orbiculatus Thunb on human hepatocellular carcinoma in an orthothopic fluorescent nude mouse model," Anticancer Research, vol.32, no. 4, pp. 12131220, 2012.

[82] M. Hu, M. Zhao, C. An et al., "Real-time imaging of apoptosis induction of human breast cancer cells by the traditional Chinese medicinal herb tubeimu," Anticancer Research, vol. 32, no. 7, pp. 2509-2514, 2012.

[83] B. Zhao, L. Liu, S. Zhao et al., "Vitexicarpin acts as a novel angiogenesis inhibitor and its target network," Evidence-Based Complementary and Alternative Medicine, vol. 2013, Article ID 278405, 13 pages, 2013.

[84] R. Albert, H. Jeong, and A. L. Barabási, "Error and attack tolerance of complex networks," Nature, vol. 406, no. 6794, pp. 378-382, 2000.

[85] H. Yu, P. M. Kim, E. Sprecher, V. Trifonov, and M. Gerstein, "The importance of bottlenecks in protein networks: correlation with gene essentiality and expression dynamics," PLoS Computational Biology, vol. 3, no. 4, pp. 713-720, 2007.

[86] W. C. Hwang, A. Zhang, and M. Ramanathan, "Identification of information flow-modulating drug targets: a novel bridging paradigm for drug discovery," Clinical Pharmacology and Therapeutics, vol. 84, no. 5, pp. 563-572, 2008.

[87] I. J. Farkas, T. Korcsmáros, I. A. Kovács et al., "Networkbased tools for the identification of novel drug targets," Science Signaling, vol. 4, no. 173, 2011. 


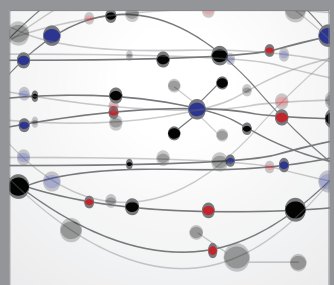

The Scientific World Journal
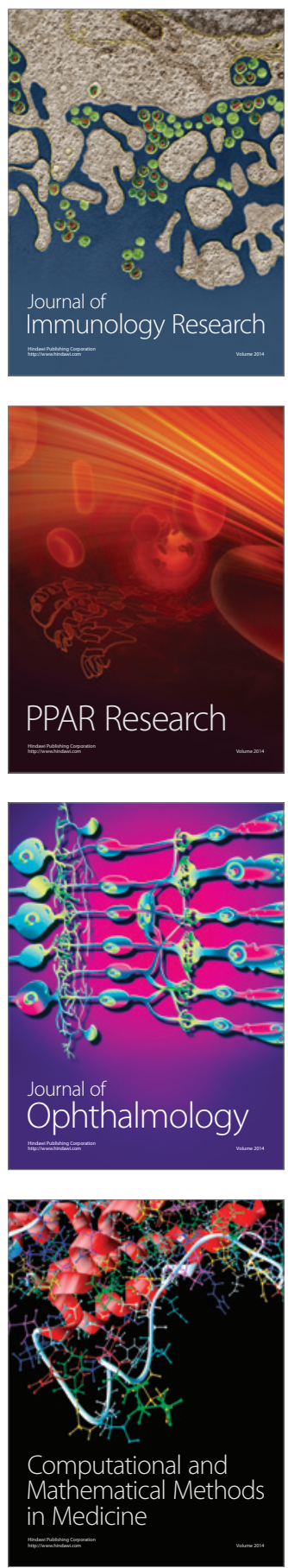

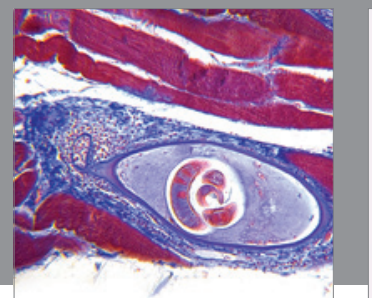

Gastroenterology

Research and Practice
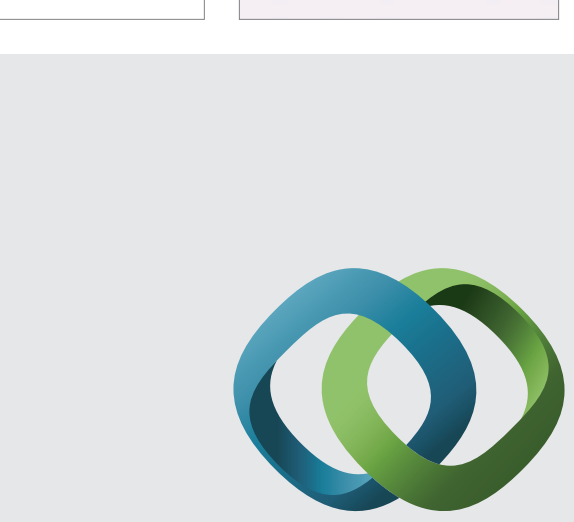

\section{Hindawi}

Submit your manuscripts at

http://www.hindawi.com
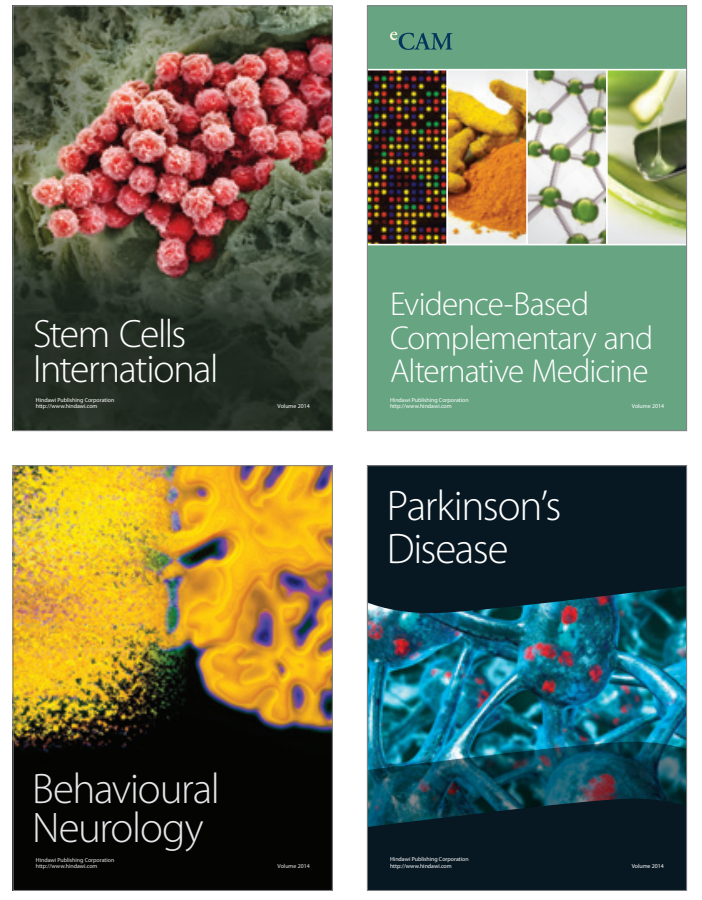
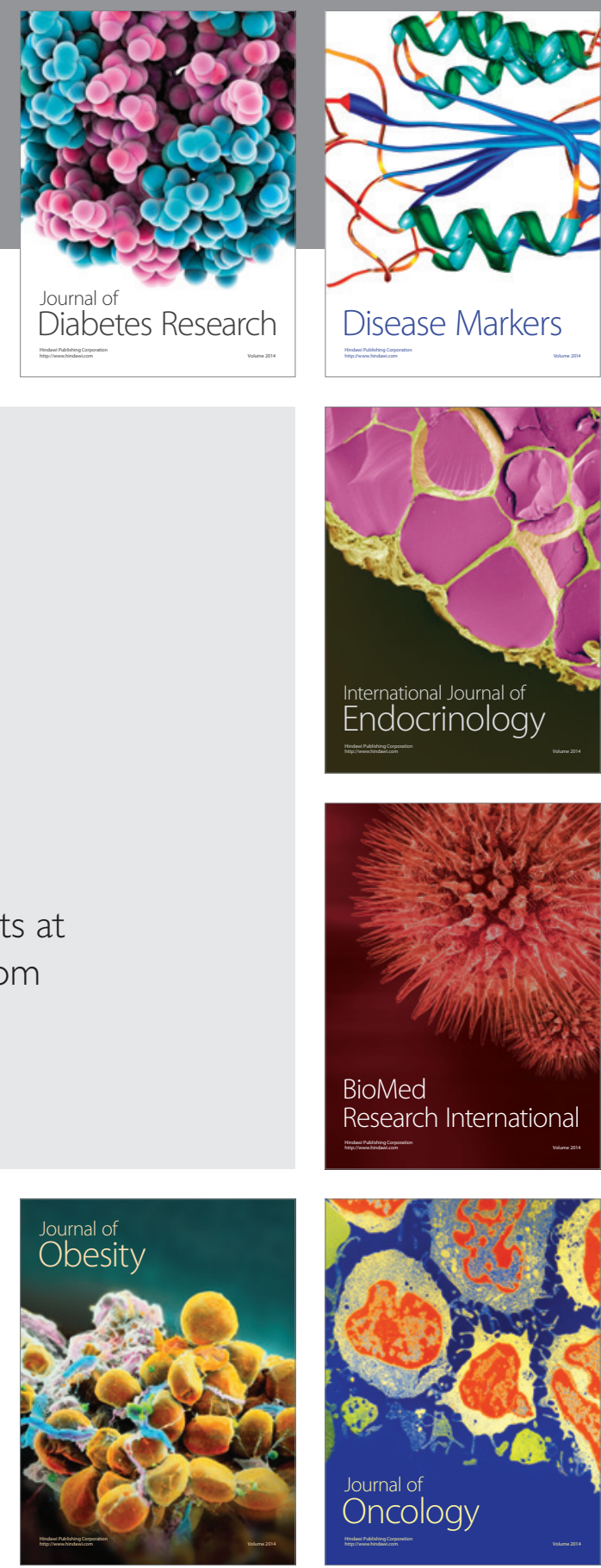

Disease Markers
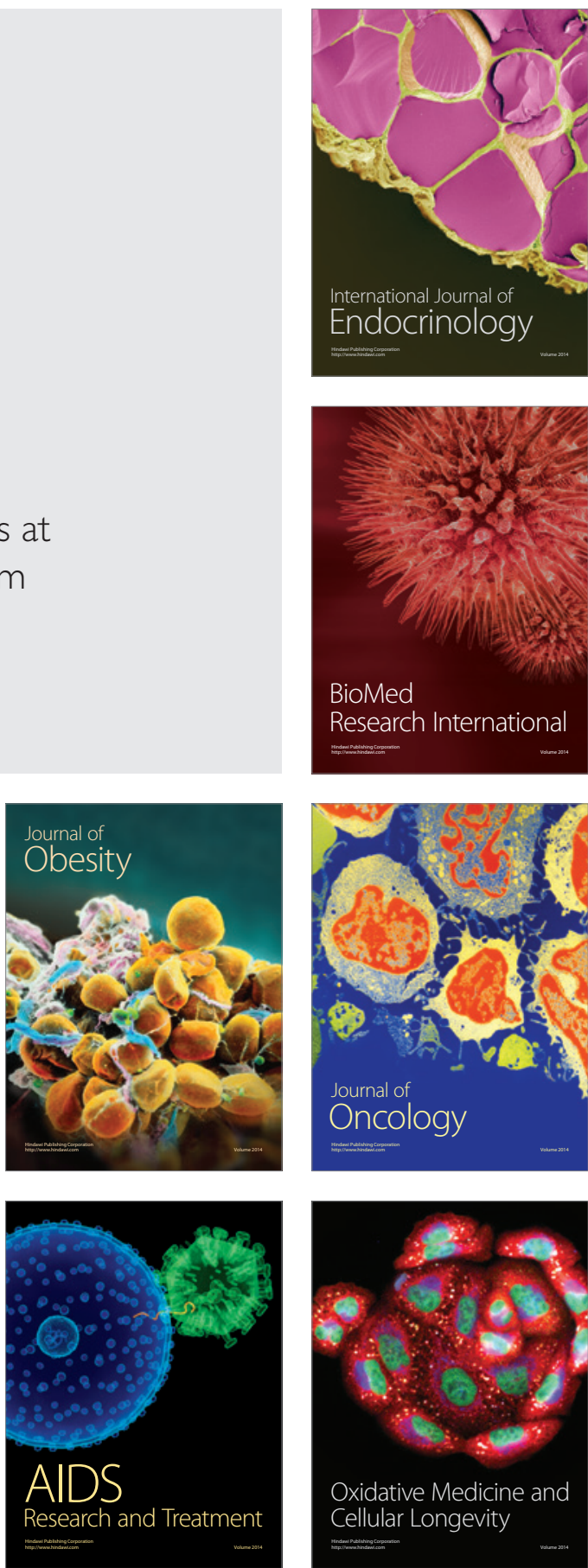